\title{
Infrared Measurements From 50 to 125 Microns
}

\author{
Earle K. Plyler and Nicolo Acquista
}

\begin{abstract}
A prism spectrometer has been changed to a long wavelength grating spectrometer by removing the prism and by replacing the Littrow mirror with a grating. Gratings of 320 and 180 lines per inch were used for the region 50 to 125 microns. A thermocouple with a crystal quartz window served as the detector. Stray radiation was greatly reduced by the use of reflection filters. Three types of reflection filters were found to be efficient. They were gratings with 2,000 lines per inch, roughened silver mirrors, and reststrahlen plates of $\mathrm{NaCl}, \mathrm{KBr}$, and $\mathrm{CsBr}$. The absorption lines of water vapor were used to calibrate the spectrometer. The spectra of several substituted ethylenes and ethanes have been measured, and absorption bands have been found between 50 and 100 microns.
\end{abstract}

\section{Introduction}

During the last 2 years prisms of CsI have been available, and it is now possible to make measurements to $50 \mu[1] .^{2}$ As quartz has its strongest reststrahlen band at $9 \mu$, it should transmit radiation at $100 \mu$. For thicknesses less than $1 \mathrm{~mm}$, quartz has a good transmission of infrared radiation in the long wavelength region. However, with prisms of crystal quartz of a refracting angle of $7.5^{\circ}$, it was found that no appreciable energy was transmitted in the region 50 to $100 \mu$. Energy was recorded in the $150-\mu$ region, but the resolution was low. Diamond is another material which is transparent in the longwavelength region, but the expense and difficulty in obtaining material of sufficient size for a prism for infrared measurements would make its use impracticable. However, windows of diamond for detectors are useful for the infrared region.

\section{Experimental Procedure}

In order to extend measurements beyond $50 \mu$, experiments were carried out by the use of small gratings. Large-grating spectrometers for the far infrared have been built in several laboratories [2], and it was felt that sufficient energy would be available with small gratings. A graing was installed in a spectrometer in place of the Littrow mirror with the prism removed. At first the observations were made with an $18^{\circ}$ off-axis collimating mirror, which was rotated a few degrees in order to fill the grating with the radiation. A small improvement in the resolution was obtained later on by installing a $14^{\circ}$ off-axis mirror. Two gratings were used in the spectrometer, which had ruled surfaces of about $2 \frac{1}{4}$ by 3 inches and were ruled with 320 lines per inch and 180 lines per inch. The spectrometer is able to accommodate slightly larger gratings, which would give an increase in energy and resolution. The grating was placed in a holder which allowed adjustments of its position, so that a mercury line in a higher order could be brought sharply in focus at the exit slit.

1 This work was done under a program of basic instrumentation, supported in part by the Office of Naval Research, Air Research and Development Command, part by the Office of Naval Research, A
and the A tomic Energy Commission.

2 Figures in brackets indicate the literature references at end of this paper.
The curvature of the entrance slit was not proper for the grating, but this did not reduce the resolution much because slits of $0.5 \mathrm{~mm}$ or larger were used for the region from 50 to $125 \mu$. However, better resolution will be obtained with straight slits. A thermocouple with a CsI window was used from 25 to $56 \mu$, and another thermocouple with a crystal quartz window was found to be suitable from 50 to $125 \mu$. It is planned to use a thermocouple with a diamond window from 25 to $125 \mu$, thus avoiding the changing: of the thermocouple for the different regions of the spectrum.

Special attention is necessary to the problem of stray radiation in the long-wavelength region. The stray radiation was reduced to a few percent by the use of reflection filters and reststrahlen plates in place of the mirrors in the spectrometer. The optical system of the grating spectrometer is shown in figure 1 after the grating had been installed and the plane mirrors replaced by reflection filters. The two small plane mirrors, immediately in front of and behind the exit slit of the spectrometer, were replaced by two plane gratings, and the plane mirror in the source box of the spectrometer was replaced by a reststrahlen plate of $\mathrm{NaCl}, \mathrm{KBr}$, or $\mathrm{CsBr}$. Also, three gratings could be used in place of the mirrors, but the reststrahlen plates are very useful in reducing second order spectra. A system which was as effective as the gratings and the reststrahlen plates in the removal of stray radiation consisted in the use of blackened silver mirrors which were first roughened with fine emory cloth. These reflection filters have been described previously [1].

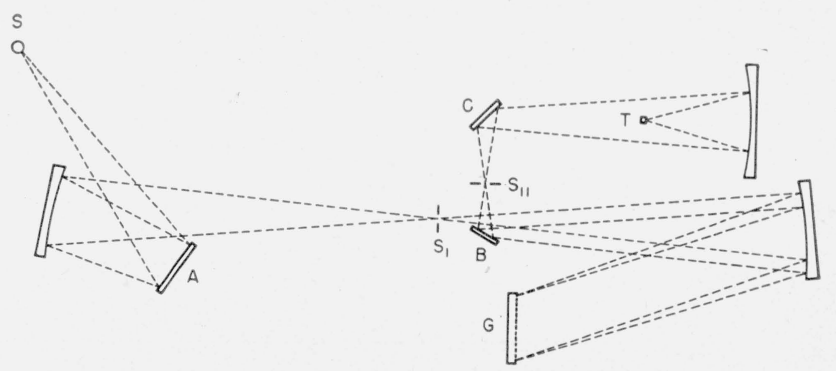

Figure 1. The optical system of the grating spectrometer. $\mathrm{A}, \mathrm{B}$, and $\mathrm{C}$ are reflection filters which have replaced the mirrors of the spectrometer. $G$ is the dispersion grating which is placed on the Littrow mirror mount, and $T$ is the thermocouple with a quartz window. 
A study has been made of the efficiency of different types of filters for removal of stray radiation. The water-vapor spectrum from 45 to $70 \mu$ was observed by the alternate use of several reflectors for the filter A of figure 1, the other parts of the spectrometer being unchanged during the comparisons. Figure 2 shows the results obtained for two gratings with 1,000 lines per inch, one roughened silver mirror, and a plate of $\mathrm{NaCl}$. The zero line represents the recorder reading when an opaque shutter is placed in the beam. The water-vapor absorption at 139 $\mathrm{cm}^{-1}$, under the atmospheric conditions of the ob- serrations, was 100 percent. By measuring how much the bottom of this absorption line is above the zero line an estimate of the stray radiation can be obtained. An estimate of the amount of the desired radiant energy reflected from the filter can be determined by measuring the difference between the deflection at the minimum of the line at $139 \mathrm{~cm}^{-1}$ and the maximum at $163 \mathrm{~cm}^{-1}$. Grating 1 is the most efficient of the four filters tested. It has 1,000 lines per inch and the plane surface was almost completely cut away in the ruling so that the central image had about 1 percent reflection. The other grating

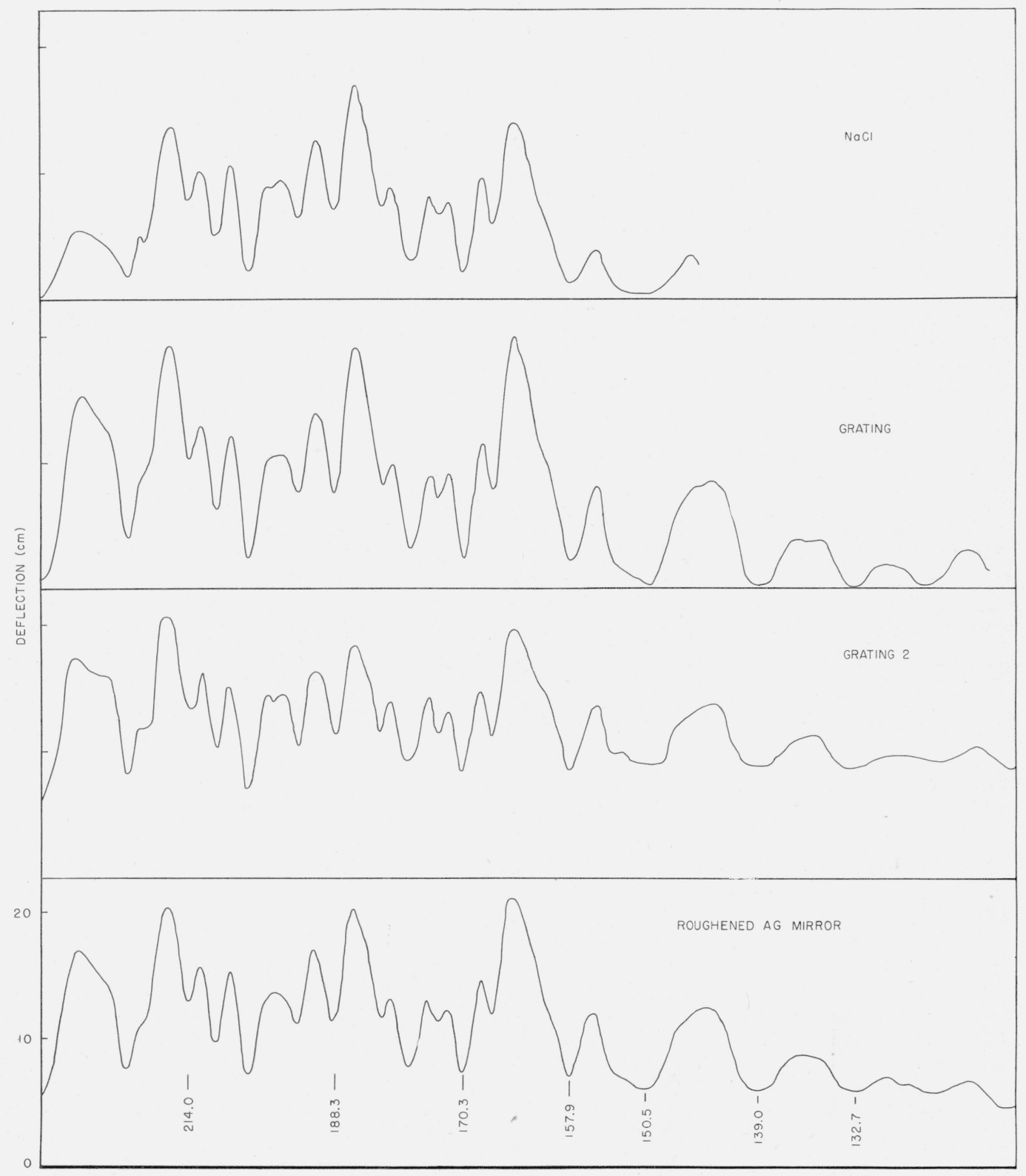

$\longleftarrow$ WAVENUMBER $\left(\mathrm{cm}^{-1}\right)$

Figure 2. A comparison of the efficiencies of different types of reflection filters.

The base line for each filter represents the deflection with an opaque shutter in the beam.

150 
(grating 11) had 1,000 lines per inch and the reflection for normal incidence was about 5 percent. Grating 11 is much less efficient in reducing the stray radiation. The roughened silver mirror does not reduce the desired energy appreciably but does allow considerable stray radiation to enter the spectrometer. The stray radiation can be made less for the silver filter by producing a greater amount of roughening on the surface, but this also reduces the desired energy. Reststrahlen plates of $\mathrm{NaCl}, \mathrm{KCl}, \mathrm{KBr}$, and $\mathrm{CsBr}$ are efficient filters for the region from 45 to $125 \mu$. The spectral region of high reflection is limited to narrow bands and one material will not cover a wide range of the infrared spectrum. The $\mathrm{CsBr}$ reststrahlen plate is useful in measurements from 100 to $125 \mu$. The second-order spectrum is intense in this region from the 180-lines-per-inch grating and it is not removed by the grating filters or the roughened silver mirror.

Since the quartz window on the thermocouple absorbed the radiation from 5 to $40 \mu$, the second order from the grating did not overlap the first order except in the region from 90 to $125 \mu$ when the roughened silver reflectors were used.

With these changes of the mirrors in the optical system just mentioned, it was found that sufficient energy for measurement could be observed from the globar source with the same amplifier that was used for the near infrared region.

The instrument has been tested and calibrated by the use of the water-vapor spectrum in the region from 25 to $125 \mu$. It has been found that the instrument can be used in three ways. First, by the use of the second and third orders of the 320- and 180lines-per-inch gratings, high resolution can be obtained in the region from 25 to $50 \mu$. Second, the 320-lines-per-inch grating, with the thermocouple having a quartz window, gives good resolution in the first order from 50 to $70 \mu$. Third, the 180lines-per-inch grating can be used from 50 to $125 \mu$. Gratings with various spacings can be used to obtain the best resolution in a particular region. One grating with 240 lines per inch would be suitable for the region from 50 to $100 \mu$.

\section{Results}

Figure 3 shows the water-vapor absorption lines from 23.5 to $33.5 \mu$ as observed with the 320 -linesper-inch grating. The top curve is the second-order

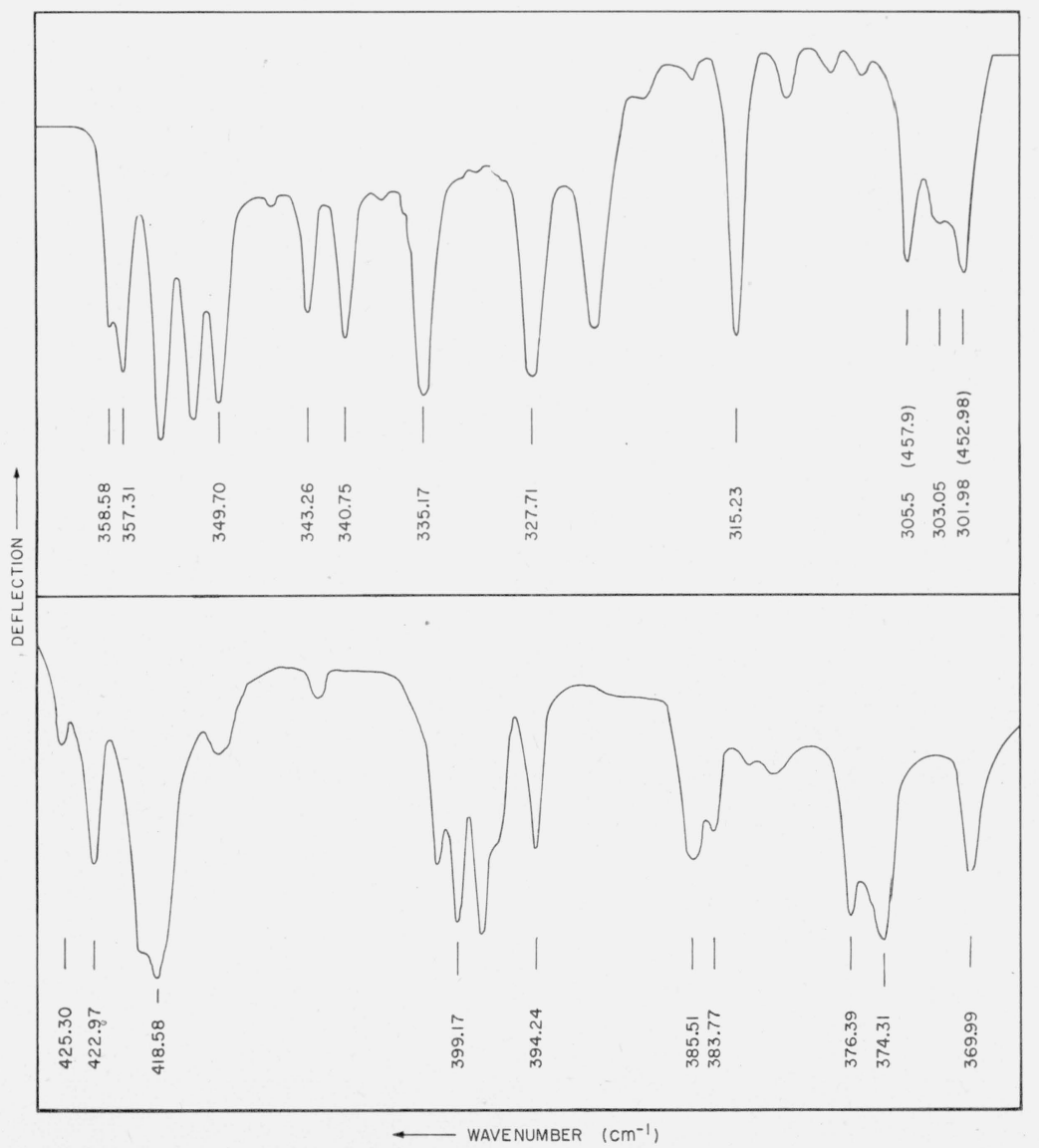

FIGURE 3. The water vapor spectrum recorded with a 320-lines-per-inch grating.

The upper curve is the second-order spectrum and the lower curve is the third-order spectrum. Overlapping of the two orders occurs at $305 \mathrm{~cm}^{-1}$. 


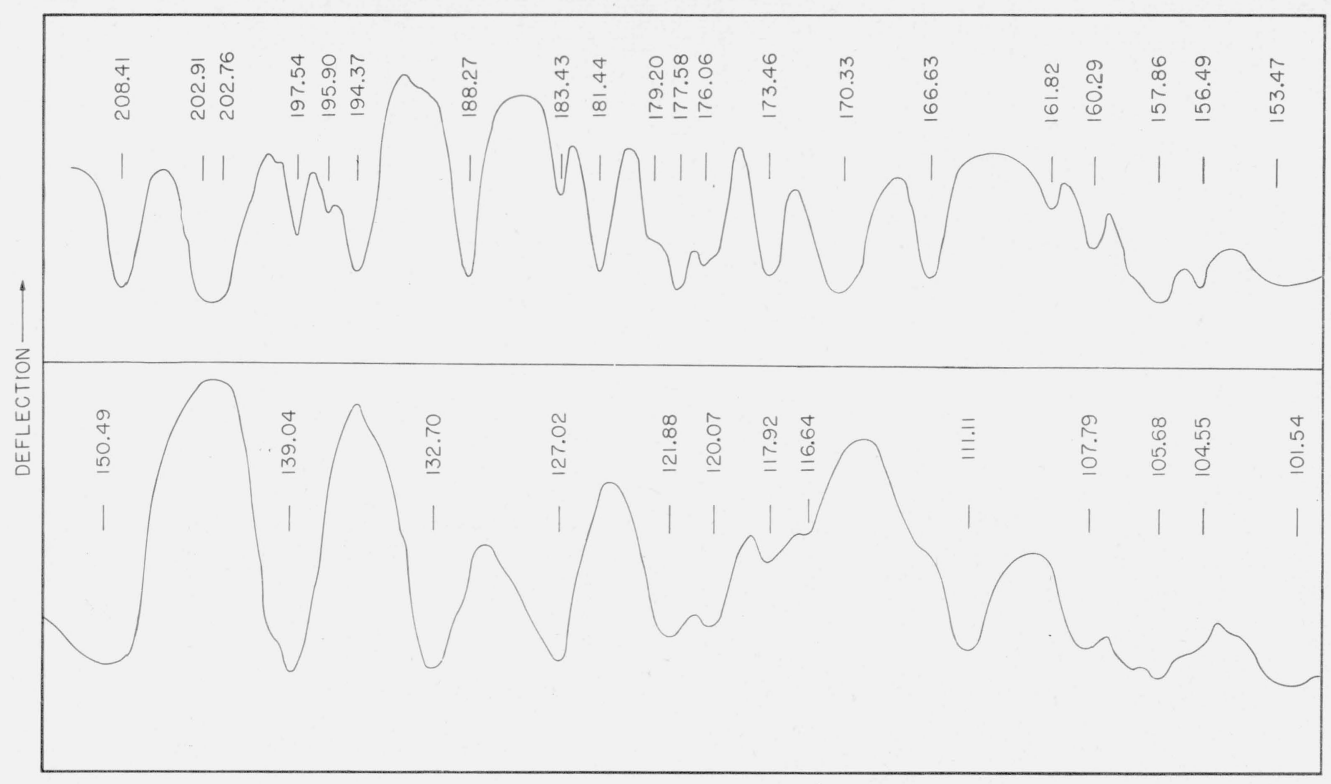

- WAVENUMBer $\left(\mathrm{cm}^{-1}\right)$

FigdRe 4. The water vapor spectrum from 100 to $200 \mathrm{~cm}^{-1}$.

The upper curve is the first-order spectrum of water vapor from 150 to $200 \mathrm{~cm}^{-1}$ as observed with the 320 -lines-per-inch grating. The lower curve is the water-vapor spectrum from 100 to $150 \mathrm{~cm}^{-1}$ as observed with a 180 -lines-per-inch grating.

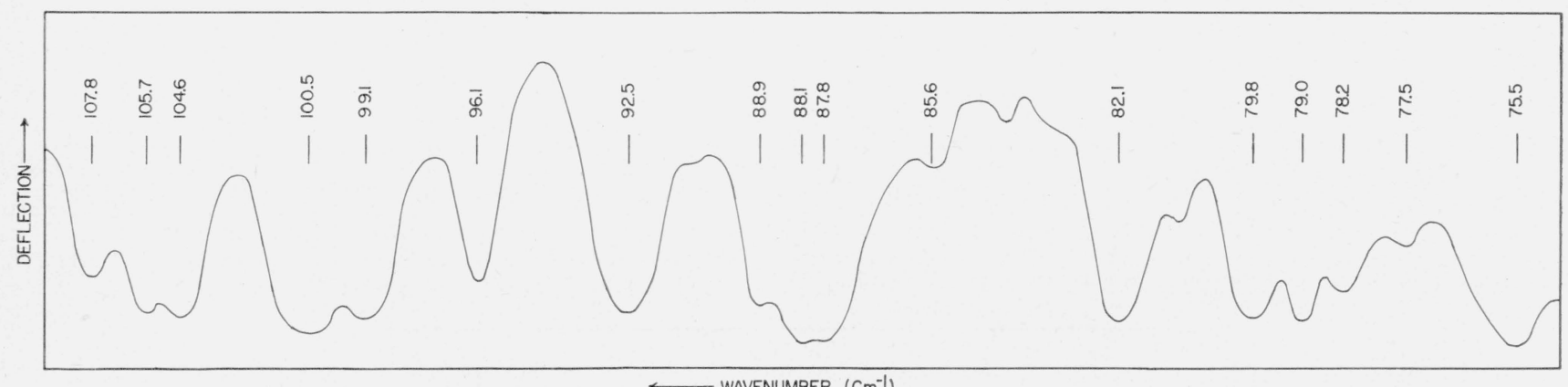

Figure 5. The absorption spectrum of $\mathrm{H}_{2} \mathrm{O}$ in the atmosphere from 75 to $100 \mathrm{~cm}^{-1}(100$ to $125 \mu)$. The observations were made with a grating of 180 lines per inch.

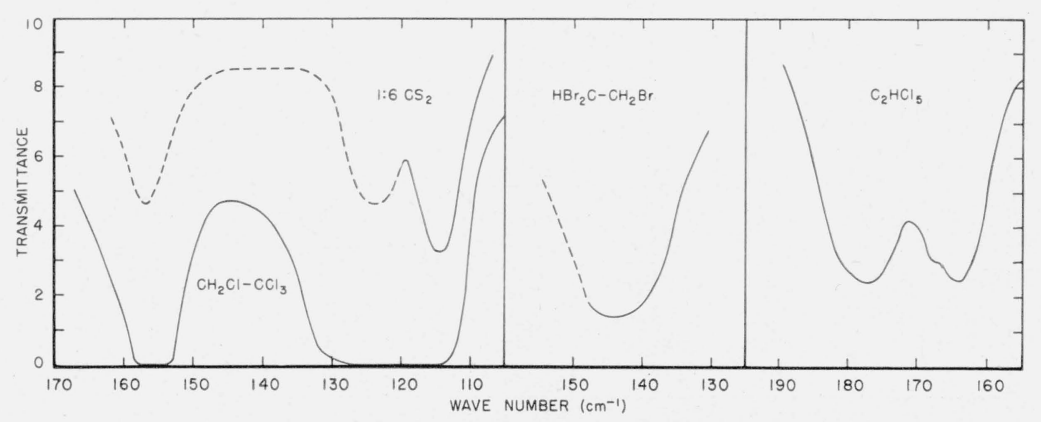

Figure 6. Absorption bands of $\mathrm{CH}_{2} \mathrm{Cl}-\mathrm{CCl}_{3}, \mathrm{HBr}_{2} \mathrm{C}-\mathrm{CH}_{2} \mathrm{Br}$, and $\mathrm{C}_{2} \mathrm{HCl}_{5}$ in the region from 110 to $190 \mathrm{~cm}^{-1}$.

The cell thickness was $1 \mathrm{~mm}$. 
spectrum, and the bottom curve is the third-order spectrum. The five lines in the region of $395 \mathrm{~cm}^{-1}$ are well resolved by the instrument. The strong absorption line at $340 \mathrm{~cm}^{-1}$ was not reported in the earlier experimental work for this region, and this transition is predicted from the energy levels involved to have appreciable intensity. In the region of $300 \mathrm{~cm}^{-1}$ the third-order spectrum is overlapped by the second-order spectrum; this overlapping can be avoided by the use of a $\mathrm{NaF}$ reflector for this region.

Figure 4, upper curve, shows the region from 50 to $65 \mu$ as observed with the 320 -lines-per-inch grating, and the lower curve is for the region from 65 to $100 \mu$ which was measured with the 180-lines-per-inch grating. Also the region from 100 to $125 \mu$, measured by the use of a $\mathrm{CsBr}$ reflector, is shown in figure 5 . Slits of $1.8 \mathrm{~mm}$ were necessary for the measurements in this region.

The lines drawn above or below the spectrum represent the positions of the water-vapor lines as calculated from their energy levels. These wavenumbers are reduced to vacuum and have been published by Benedict, Claassen, and Shaw [3]. Only strong single lines have been used for the calibration, but the approximate positions of some complex lines are also listed on the figure. The lines were identified by using the wavenumbers obtained from the grating constant. While the water-vapor spectrum is of great value for calibration and testing of the instrument, it should be removed for the measurement of other spectra. This can be best accomplished by placing the spectrometer in a vacuum chamber. The absorption of the water vapor was greatly reduced by circulating dry nitrogen in the enclosure of the spectrometer. In the measurement of the absorption bands of liquids and vapors the removal of the water-vapor spectrum is essential to obtain band shapes.

The primary purpose of developing this instrument was to have available a spectrometer for measuring the absorption spectra of materials in the region from 50 to $125 \mu$. In the study of the vibrational bands of many molecules it was found that it was difficult to make a complete assignment of the different modes of vibration because several of the bending vibrations would be between 50 and $125 \mu$. Since the instrument has been in operation the long- wavelength spectra of $\mathrm{C}_{2} \mathrm{Cl}_{4}, \mathrm{C}_{2} \mathrm{Br}_{4}, \mathrm{C}_{2} \mathrm{~F}_{3} \mathrm{Br}$ have been measured and their spectra analyzed. Cells with windows of crystal quartz, $0.2 \mathrm{~mm}$ in thickness, were used for these measurements. Many of the spectra of the substituted ethylenes, ethanes, and benzenes which contain fluorine, chlorine, bromine, or iodine will contain bands beyond $50 \mu$, and an instrument of this type will be of great value in obtaining sufficient data for a complete vibrational analysis. As an example of measurements on longwavelength spectra figure 6 shows the absorption bands of $\mathrm{CH}_{2} \mathrm{Cl}-\mathrm{CCl}_{3}, \mathrm{CHBr}_{2}-\mathrm{CH}_{2} \mathrm{Br}$, and $\mathrm{C}_{2} \mathrm{HCl}_{5}$ between 50 and $100 \mu$. The longest wavelength band which was observed for these materials appeared at $114 \mathrm{~cm}^{-1}$. With a cell of $1 \mathrm{~mm}$ thickness it was necessary to dilute $\mathrm{CH}_{2} \mathrm{Cl}-\mathrm{CCl}_{3}$ with carbon disulfide in ratio 1 part to 6 so that the bands could be resolved.

The results that have been obtained demonstrate that spectra can be measured in the region from 50 to $125 \mu$ with small gratings. For the arrangement with one grating of 200 to 240 lines per inch and a thermocouple with a quartz window, the costs should not be greater than $\$ 1,000$. Where several gratings are employed and a vacuum enclosure with the necessary external controls is made, the cost would be greater. The number of gratings which will be employed and the changes in the filters for different regions will of necessity depend on the nature of the measurements which are being made. For the measurement of vibrational spectra and the transmittances of solids and liquids a single grating is adequate.

\section{References}

¡1] N. Acquista and Earle K. Plyler, J. Opt. Soc. Am. 43, 977 (1953).

[2] H. M. Randall, Rev. Sci. Instr. 3, 196 (1932); T. King McCubbin and Wm. M. Sinton, J. Opt. Soc. Am. 40, 537 (1950) ; R. A. Oetjen, W. H. Haynie, W. M. Ward, R. L. Hansler, H. E. Schauwecker, and E. E. Bell, J. Opt. Soc. Am. 42, 559 (1952); L. Genzel and W. Eckhardt, Z. Physik 139, 578 (1954).

[3] W. S. Benedict, H. H. Claassen, and J. H. Shaw, J, Research NBS 49, 91 (1952) RP2347.

Washington, November 30, 1955. 